\title{
Electromagnetic Waves Absorption by Graphene-Magnetic Semiconductor Multilayered Nanostructure in External Magnetic Field: Voight Geometry
}

\author{
D. KuZMin ${ }^{a}, *$ I. BYCHKOV ${ }^{a}$ AND V. SHAVROV ${ }^{b}$ \\ ${ }^{a}$ Chelyabinsk State University, Br. Kashirinykh 129, 454001 Chelyabinsk, Russia \\ ${ }^{b}$ Kotelnikov Institute of Radio-Engineering and Electronics of RAS, Mokhovaya 11/7, 125009 Moscow, Russia
}

\begin{abstract}
Absorption of electromagnetic waves by graphene-magnetic semiconductor multilayered structure has been investigated for the Voight geometry with taking into account the dissipation processes. The possibility of control of electrodynamic properties of the structure by an external magnetic field, changing in number of periods of the structure, chemical potential of the graphene layers and temperature has been shown. Electrodynamics of the structure is also sensitive to polarization of the incident wave. The structure shows to be especially controllable at terahertz frequencies.
\end{abstract}

DOI: 10.12693/APhysPolA.127.528

PACS: 41.20.Jb, 42.25.Bs, 68.65.Ac, 68.65.Cd, 68.65.Pq

\section{Introduction}

Graphene was firstly produced about ten years ago [1]. Since then and to nowadays graphene continues to surprise researchers by its unusual physical properties. High mobility of charge carriers makes it a promising material for use in various applications, in particular, as a basis for wide range of nanoelectronic devices [2]. Graphene layer can support highly localized surface electromagnetic waves - surface plasmon polaritons [3, 4], that caused the possibility to use it for various plasmonic applications. Recently, hyperbolic metamaterial on basis of graphene-dielectric superlattice has been proposed and studied [5,6]. Replacement of dielectric in graphenebased superlattices by materials with a more complex electrodynamic properties can lead to greater controllability of electrodynamics of the structure. So, for example, metamaterial composed of periodic stacking of graphene-liquid crystal layers has been recently proposed for far-infrared frequencies [7]. The optical parameters of such a structure can be controlled by tuning of permeability of the liquid crystal and the surface conductivity of the graphene sheets. The metamaterial is able to show both the elliptic and hyperbolic dispersion. Some aspects of tuning of electromagnetic waves absorption, reflection and transmission of graphene-magnetic semiconductor multilayered structure by external magnetic field directed perpendicular to the periodicity axis of the structure (the Faraday geometry) have been investigated in [8]. Frequency dispersion of the permittivity is one of the significant differences of semiconductor from dielectrics; the plasma waves can be excited in the semiconductor structures; the helicons can propagate in the material

${ }^{*}$ corresponding author; e-mail: kuzminda89@gmail.com along an external magnetic field direction. The magnetic semiconductors have a number of specific features: they may have a large magnetoresistance [9], magnetooptical properties [10], the mutual interaction of the spin and plasma subsystems [11], etc. Due to peculiarities of electromagnetic waves propagation along the external magnetic field in the structure graphene-magnetic semiconductor, it is interesting to study the propagation of electromagnetic waves for other directions of the magnetic field. This work is devoted to investigation of electrodynamic characteristics of graphene-magnetic semiconductor multilayered structure for the Voight geometry, i.e. when an external magnetic field is directed perpendicular to the periodic axis of the structure.

\section{Geometry of the problem}

Geometry of the problem is represented in Fig. 1. The structure is a periodic stacking of graphene monolayers and magnetic semiconductor slabs. Suppose that linearly polarized plane electromagnetic wave is normally incident in the surface of the structure and that an external magnetic field $\boldsymbol{H}_{0}$ is directed perpendicular to the periodicity axis of the structure (i.e. the Voight geometry). The coordinate axes are chosen so that the $z$ axis coincides with the direction of the external magnetic field, the $y$ axis coincides with the periodicity axis of the structure. The thickness of the magnetic semiconductor is denoted $d ; D$ is the thickness of the whole structure. The symmetry of the problem is broken by external magnetic field, so, electrodynamic properties of the structure will depend on the polarization of the incident electromagnetic wave. The angle between the $z$ axis and the polarization of the electric component of the electromagnetic wave will be denoted $\theta$.

For solving this problem, one has to know the characteristics of each component of the structure. For the magnetic semiconductor such characteristics are the tensors 


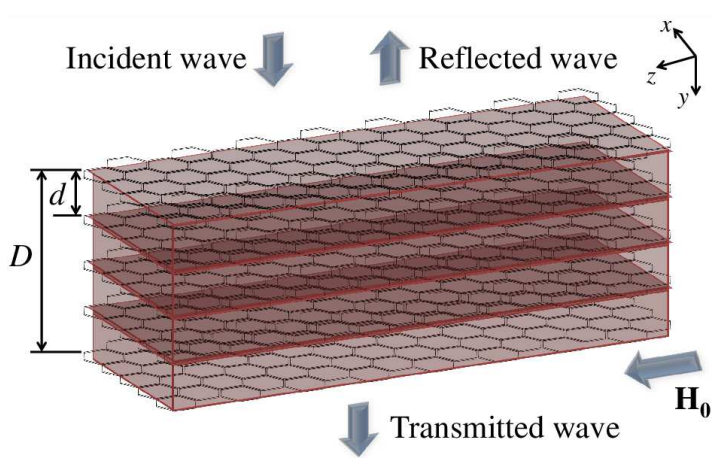

Fig. 1. Geometry of the structure with the thickness $D$ composed of a periodic structure of graphene layers separated by magnetic semiconductor slabs with the thickness $d$ placed in external magnetic field $\boldsymbol{H}_{\mathbf{0}}$.

of the permeability $\hat{\mu}$ and the permittivity $\hat{\varepsilon}$. The forms of the tensors are characteristic of an isotropic, magnetized ferromagnet and an isotropic, magnetized, singlecomponent plasma in a semiconductor $[12,13]$ :

$$
\begin{aligned}
& \hat{\mu}=\left(\begin{array}{ccc}
\mu_{\perp} & \mathrm{i} \mu_{a} & 0 \\
-\mathrm{i} \mu_{a} & \mu_{\perp} & 0 \\
0 & 0 & \mu_{\|}
\end{array}\right) \text {; } \\
& \mu_{\perp}=1+\frac{\omega_{M}\left(\omega_{H}-\mathrm{i} \alpha \omega\right)}{\omega_{H}^{2}-\left(1+\alpha^{2}\right) \omega^{2}-2 \mathrm{i} \alpha \omega \omega_{H}} ; \\
& \mu_{a}=\frac{-\omega_{M} \omega}{\omega_{H}^{2}-\left(1+\alpha^{2}\right) \omega^{2}-2 \mathrm{i} \alpha \omega \omega_{H}} ; \\
& \mu_{\|}=1-\frac{\mathrm{i} \alpha \omega_{M}}{\omega+\mathrm{i} \alpha \omega_{H}} \text {. } \\
& \hat{\varepsilon}=\left(\begin{array}{ccc}
\varepsilon_{\perp} & \mathrm{i} \varepsilon_{a} & 0 \\
-\mathrm{i} \varepsilon_{a} & \varepsilon_{\perp} & 0 \\
0 & 0 & \varepsilon_{\|}
\end{array}\right) \text {; } \\
& \varepsilon_{\perp}=\varepsilon_{0}\left(1-\frac{\omega_{\mathrm{p}}^{2}(\omega+\mathrm{i} \nu)}{\omega\left[(\omega+\mathrm{i} \nu)^{2}-\omega_{\mathrm{c}}^{2}\right]}\right) \\
& \varepsilon_{a}=\varepsilon_{0} \frac{\omega_{\mathrm{p}}^{2} \omega_{\mathrm{c}}}{\omega\left[(\omega+\mathrm{i} \nu)^{2}-\omega_{\mathrm{c}}^{2}\right]} ; \\
& \varepsilon_{\|}=\varepsilon_{0}\left(1-\frac{\omega_{\mathrm{p}}^{2}}{\omega(\omega+\mathrm{i} \nu)}\right) \text {. }
\end{aligned}
$$

Here, $\omega_{H}=g H_{0}, \omega_{M}=4 \pi g M_{0}, g$ is the gyromagnetic ratio, $M_{0}$ is the saturation magnetization, $\alpha$ is the damping parameter, $\varepsilon_{0}$ is the lattice caused part of the permittivity, $\omega_{\mathrm{p}}=\sqrt{4 \pi n_{s} e^{2} / m^{*}}$ and $\omega_{\mathrm{c}}=e H_{0} / m^{*} c$ are the plasma and the cyclotron frequencies, consequently, $e$ and $m^{*}$ are the charge and the effective mass of carriers, $n_{s}$ is the carriers density, $\nu$ is the effective collision rate.

Graphene can be represented as a conductive surface [3] with the frequency dependent conductivity $\sigma(\omega)=\sigma^{\text {intra }}(\omega)+\sigma^{\text {inter }}(\omega)$, which has been obtained in [14]:

$$
\begin{aligned}
& \sigma^{\text {intra }}(\omega)=\frac{2 \mathrm{i} e^{2}}{\hbar \pi} \frac{k_{\mathrm{B}} T}{(\hbar \omega-\mathrm{i} \Gamma)} \ln \left(2 \cosh \left(\frac{\mu_{\mathrm{ch}}}{2 k_{\mathrm{B}} T}\right)\right) \\
& \sigma^{\text {inter }}(\omega)=\frac{e^{2}}{4 \hbar}\left[\frac{1}{2}+\frac{1}{\pi} \arctan \left(\frac{(\hbar \omega-\mathrm{i} \Gamma)-2 \mu_{\mathrm{ch}}}{2 k_{\mathrm{B}} T}\right)\right] \\
&-\frac{\mathrm{i}}{2 \pi} \ln \left(\frac{\left[(\hbar \omega-\mathrm{i} \Gamma)+2 \mu_{\mathrm{ch}}\right]^{2}}{\left[(\hbar \omega-\mathrm{i} \Gamma)-2 \mu_{\mathrm{ch}}\right]^{2}+4 k_{\mathrm{B}}^{2} T^{2}}\right)
\end{aligned}
$$

Here, $\mu_{\mathrm{ch}}$ is a chemical potential of graphene, $\Gamma / \hbar$ is the scattering rate. In chosen geometry, conductivity of graphene not depends on the magnetic field value.

For solving the problem one has to use the system of Maxwell's equations

$$
\operatorname{rot} \boldsymbol{E}=-c^{-1} \partial \boldsymbol{B} / \partial t ; \quad \operatorname{rot} \boldsymbol{H}=c^{-1} \partial \boldsymbol{D} \partial t
$$

with the material equations

$$
\boldsymbol{D}=\hat{\varepsilon} \boldsymbol{E} ; \quad \boldsymbol{B}=\hat{\mu} \boldsymbol{H} ; \quad \boldsymbol{j}=\hat{\sigma} \boldsymbol{E}
$$

and the boundary conditions

$$
\left(\boldsymbol{E}_{2}-\boldsymbol{E}_{1}\right) \times \boldsymbol{n}_{12}=0 ; \quad\left(\boldsymbol{H}_{2}-\boldsymbol{H}_{1}\right) \times \boldsymbol{n}_{12}=4 \pi \boldsymbol{j} / c,
$$

where indexes 1 and 2 mean the fields in the first and the second medium, $\boldsymbol{n}_{12}$ is the normal vector to the partition surface directed from the first medium to the second one, $\boldsymbol{j}$ is the density of the surface current in graphene layer.

Solving the system of Eqs. (4)-(6) for each layer of magnetic semiconductor we obtain the amplitudes of reflected and transmitted waves. Then reflectance $R$ and transmittance $T$ can be found

$$
R=\frac{\left|E_{x R}\right|^{2}+\left|E_{z R}\right|^{2}}{\left|E_{x 0}\right|^{2}+\left|E_{z 0}\right|^{2}} ; \quad T=\frac{\left|E_{x T}\right|^{2}+\left|E_{z T}\right|^{2}}{\left|E_{x 0}\right|^{2}+\left|E_{z 0}\right|^{2}},
$$

where indexes $R$ and $T$ denote amplitudes of reflected and transmitted waves, consequently, index " 0 " denotes amplitude of incident wave. Obtaining their values we define the absorptance $A=1-R-T$.

For the numerical simulation we will use the characteristic parameters of magnetic semiconductor $\mathrm{CdCr}_{2} \mathrm{Se}_{4}$ with the Curie temperature $T_{\mathrm{C}}=130 \mathrm{~K}[15,16]$ :

$$
\begin{aligned}
& M_{0}=350 \mathrm{G}, \alpha=0.1, g=1.75 \times 10^{7} \mathrm{Oe}^{-1} \mathrm{~s}^{-1}, \\
& \varepsilon_{0}=20, m^{*}=0.15 m_{e}, n_{s}=10^{18} \mathrm{~cm}^{-3}, \\
& \nu=10^{15} \mathrm{~s}^{-1} .
\end{aligned}
$$

For simulation of the graphene properties we will use parameters $v_{F}=10^{8} \mathrm{~cm} / \mathrm{s}, \Gamma=2 \times 10^{-15} \mathrm{erg}$, value of the chemical potential $\mu_{\mathrm{ch}}$ depends on temperature $T$ and carrier density in graphene $n_{0}$. How it has been shown in [14], for temperatures $T \approx 10 \mathrm{~K}$ and carrier density $n_{0} \approx 10^{11} \mathrm{~cm}^{-2}$ value of chemical potential is $\mu_{\mathrm{ch}} \approx 1 \times 10^{-14} \mathrm{erg}$. The carrier density can be changed either by doping or with the help of a constant electric field.

\section{Results and discussion}

Due to resonant dependences of components of permittivity, permeability tensors of magnetic semiconductor (1), (2) and conductivity of graphene (3), it is clear that reflectance, transmittance and absorptance will have some features near the resonant. The calculations show (see Fig. 2) that electrodynamic characteristics of the 


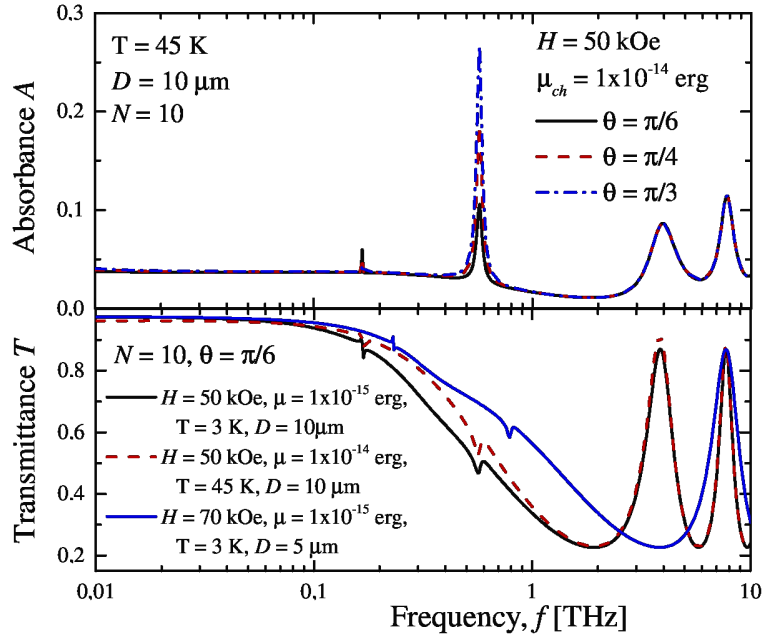

Fig. 2. Frequency dependences of electromagnetic waves transmittance $T$, and absorptance $A$ for different parameters of the structure. $N$ is a number of periods, $f=\omega / 2 \pi$.

structure depend on the polarization of an incident wave. For electromagnetic wave polarized along magnetic field direction $(\theta=0)$, there is no resonance in absorptance associated with the ferromagnetic resonance of the magnetic semiconductor, and a cyclotron associated resonance has a maximum. Otherwise, when electromagnetic wave is polarized perpendicular to the magnetic field direction $(\theta=\pi / 2)$, there is no a cyclotron associated resonance, and a ferromagnetic associated resonance has a maximum. Electrodynamic characteristics of the structure also depend on external magnetic field value. Increase in magnetic field leads to increase in ferromagnetic and cyclotron associated resonant frequencies. Change in temperature of the structure leads to change in transmittance and decrease in absorptance of electromagnetic waves in frequency range of $0.1-1 \mathrm{THz}$. Increase in number of the graphene layers leads to increase in absorptance (see Fig. 3). It is well known [14] that graphene

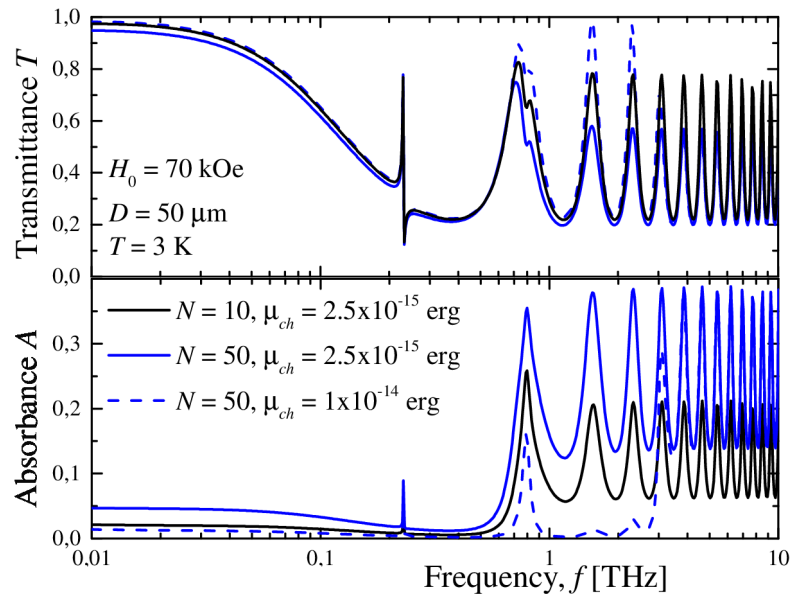

Fig. 3. Frequency dependencies of electromagnetic waves transmittance $T$, and absorptance $A$ for different number of periods $N$ and chemical potential, $f=\omega / 2 \pi$. has a logarithmic singularity at the interband absorption threshold $\omega=2 \mu_{\mathrm{ch}} / \hbar$. This leads to corresponding increase in absorptance at frequencies more than $2 \mu_{\mathrm{ch}} / \hbar$. The chemical potential can be changed, for example, by applying of external electric field. So, increase in chemical potential value leads to shift of the interband absorption threshold to higher frequencies. It is possible to get more strong absorption of electromagnetic waves by tuning the chemical potential, especially at ferromagnetic, cyclotron or size resonances.

\section{Acknowledgments}

This work was supported by grants of RFBR \# 13-0700462 and RScF \# 14-22-00279.

\section{References}

[1] K.S. Novoselov, A.K. Geim, S.V. Morozov, D. Jiang, Y. Zhang, S.V. Dubonos, I.V. Grigorieva, A.A. Firsov, Science 306, 666 (2004).

[2] Zh. Chen, Yu.-M. Lin, M.J. Rooks, Ph. Avouris, Physica E 40, 228 (2007).

[3] S.A. Mikhailov, K. Ziegler, Phys. Rev. Lett. 99, 016803 (2007).

[4] Yu.V. Bludov, M.I. Vasilevskiy, N.M.R. Peres, Eur. Phys. Lett. 92, 68001 (2010).

[5] I.V. Iorsh, I.S. Mukhin, I.V. Shadrivov, P.A. Belov, Yu.S. Kivshar, Phys. Rev. B 87, 075416 (2013).

[6] M.A. Othman, C. Guclu, F. Capolino, Opt. Expr. 25, 7614 (2013).

[7] A. Madani, S. Zhong, H. Tajalli, S. Roshan Entezar, A. Namdar, Y. Ma, Prog. Electromagn. Res. 143, 545 (2013).

[8] D.A. Kuzmin, I.V. Bychkov, V.G. Shavrov, IEEE Trans. Magn. 50, 1 (2014).

[9] Y.D. Park, A. Wilson, A.T. Hanbicki, J.E. Mattson, T. Ambrose, G. Spanos, B.T. Jonker, Appl. Phys. Lett. 78, 2739 (2001).

[10] A.M. Shuvaev, G.V. Astakhov, A. Pimenov, C. Brne, H. Buhmann, L.W. Molenkamp, Phys. Rev. Lett. 106, 107404 (2011).

[11] N.P. Demchenko, L.I. Kats, I.S. Nefedov, M.Yu. Zharkov, Radiophys. Quantum Electron. 32, 668 (1989).

[12] A.G. Gurevich, G.A. Melkov, Magnetization Oscillations and Waves, CRC, New York 1996.

[13] F.G. Bass, A.A. Bulgukov, Kinetic and Electrodynamic Phenomena in Classical and Quantum Semiconductor Superlattices, Nova Sci. Publ., New York 1997.

[14] L.A. Falkovsky, Phys. Usp. 51, 887 (2008).

[15] A.G. Gurevich, Ju.M. Jakovlev, V.I. Karpovich, A.N. Ageev, E.V. Rubalskaja, Phys. Lett. A $\mathbf{4 0}$, 69 (1978).

[16] H.W. Lehmann, G. Harbeke, J. Appl. Phys. 38, 946 (1967). 